\title{
SUPERFICIAL PUNCTATE KERATITIS
}

BY

Lieut.-Col. R. E. Wright, C.I.E., M.D., I.M.S.

PROFESSOR OF OPHTHALMOLOGY, MEDICAL COLLEGE, AND SUPERINTENDENT, GOVERNMENT OPHTHALMIC HOSPITAL, MADRAS

AND CO-WORKERS

Continued

THE epidemic described in our previous communication continued with increasing intensity in the second half of 1929 , so that by the end of the year some 3,500 cases had come under our observation. The monthly incidence of cases reporting at the hospital is shown in the chart. It will be seen that by February, 1930, the epidemic had practically disappeared.

The character of the disease appeared to change considerably as time went on, so that there was no doubt that the external signs were very much more severe than they had been in the early part of the epidemic. In the latter part of 1929 , it was not infrequent to observe patients with exceedingly red eyes, the conjunctival

Chart showing the monthly incidence of new cases of Superficial punctate heratitis reporting at the Hospital.

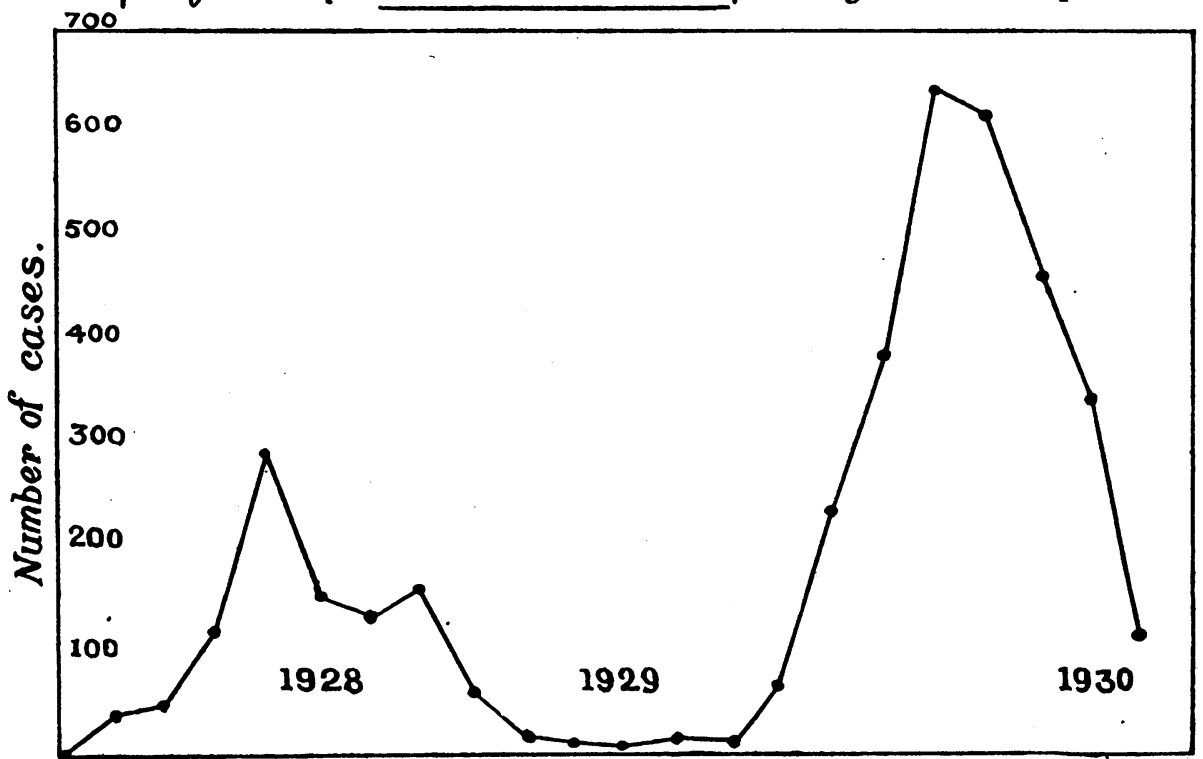

June July Aug Sep Oct Nor Der. Jan Feb Mar Apl. May June Julg Aug Sep. Oct. Nor Dec Jan Feb 
inflammation resembling that which might result from the use of irritants. It almost appeared as if frequent passage in man had intensified the virulence of the virus. On discussing this aspect of the epidemic with Lt.-Col. H. H. King, I.M.S., Director, King Institute of Preventive Medicine, Guindy, he regarded it as a distinct possibility, and when presented with cases in bulk at the beginning of 1930, he noticed a distinct difference in the severity of the external signs as compared with cases shown to him in 1928. At the former time it would have been very easy to miss the conjunctival signs altogether, and no doubt they frequently were missed, so that it is easy to understand why this disease was often first noticed in the out-patient department in the macular stage. We were able to demonstrate to him in a single morning some twenty cases which formerly would have been classed as Kirkpatrick's macular keratitis, but which had been under observation in our out-patient department from the time of onset with an initial conjunctival hyperaemia.

With regard to our original communication, Col. King points out some statistical omissions which may be mentioned here.

The table showing influence of age and sex ought to have given expected percentages of attacks thus :-

\begin{tabular}{|c|c|c|c|c|c|c|c|c|}
\hline \multirow{2}{*}{ Sex } & \multicolumn{7}{|c|}{ Age Period } & \multirow{2}{*}{ Total. } \\
\hline & $1-9$ & $10-19$ & $20-29$ & $30-39$ & $40-49$ & $50-59$ & $60-69$ & \\
\hline Males ... & 3 & 113 & 413 & 167 & 55 & 16 & 5 & 772 \\
\hline \multirow[t]{2}{*}{ Females ... } & 4 & 43 & 55 & 27 & 9 & 11 & 2 & 151 \\
\hline & 7 & 156 & 468 & 194 & 64 & 27 & 7 & \multirow[t]{4}{*}{923} \\
\hline Percentage & 0.76 & 16.9 & 50.7 & $21 \cdot 02$ & 6.93 & $2 \cdot 93$ & 0.76 & \\
\hline Expected* & 20.9 & $19 \cdot 3$ & $21 \cdot 2$ & $16 \cdot 2$ & 111 & 6.6 & $4 \cdot 7$ & \\
\hline$\underset{\text { Expected }}{\text { Observed }}$ & .036 & 0.87 & $2 \cdot 39$ & $1 \cdot 3$ & 0.62 & 0.44 & 0.16 & \\
\hline
\end{tabular}

"Expected, if the distribution were uniform for all ages, $i . e .$, if the age distribution were the same as the census proportion of people living at different ages.

It will be seen that there is a very definite and marked escape at early ages. This is the most striking thing about the age distribution. 
The sex distribution may be better shown thus :-

\begin{tabular}{|c|c|c|c|c|c|}
\hline \multicolumn{3}{|c|}{ Sex } & $\begin{array}{l}\text { Percentage } \\
\text { observed }\end{array}$ & $\begin{array}{l}\text { Percentage } \\
\text { expected }\end{array}$ & Ratio $\frac{\text { Observed }}{\text { Expected }}$ \\
\hline Males & $\ldots$ & $\ldots$ & $83 \cdot 6$ & 64.3 & $1 \cdot 27$ \\
\hline Females & $\ldots$ & $\ldots$ & $16 \cdot 4$ & $35 \cdot 7$ & 0.46 \\
\hline
\end{tabular}

* Expected, if the proportion amongst males and females were the same as the general proportion of males and females attending the hospital, namely (1.8 to 1.)

Males were evidently more liable to attack than females.

Again, the human subjects chosen for experimental transmission were actually in-patients in the hospital. It is desirable, therefore, to state the frequency with which superficial punctate keratitis appeared amongst other hospital patients during the time these experimental cases were under observation. Of this we have no record, but we know that amongst some sixty members of the staff living under similar conditions there were no attacks in the same period.

- A number of further observations were recorded, some of which are new, and it will be convenient to deal with these under separate headings.

\section{Incubation Period}

In spite of the large number of cases it was difficult to get accurate figures as to the period of incubation under natural conditions. Our experimental work appeared to show that it was somewhere between three and nine days. In the relatively few cases collected of infections under natural conditions, in which we could be certain of our facts, that is to say, in which cases arrived in the epidemic area from a free area, or left the epidemic area for a free area, the range appeared to be between one week and 34 days. Several cases of approximately a month were observed.

\section{Acquired Immunity}

We were at first under the impression that one attack gave protection against a second, and it was so stated in our original paper. It later became apparent that this was not so, and during the latter part of the epidemic we frequently observed an attack occurring in the second eye at a comparatively short interval after the attack in the first eye. In several cases there was an interval of a month or so, in others the interval was longer. We recorded, moreover, two instances in which there was a second attack in the same eye, in one case within two months and in two cases after an interval of a year. The former may perhaps be regarded as a recurrence, but recurrences in the ordinary sense of the term did 
not constitute a feature of the disease. Exacerbations were not uncommon, e.g., a relatively mild conjunctival hyperaemia showing improvement, might be followed by a renewal of symptoms with the appearance of spots at the corneal periphery, which in turn becoming quiescent might be followed by a further corneal extension with fresh spots and symptoms.

\section{Greater Frequency of Attack in One Eye}

It was at one time surmised that the left eye was more frequently attacked than the right; this was so in actual fact for a time during the earlier stages of the epidemic. On working out the figures for a consecutive 2,000 cases observed towards the latter part of the epidemic, it appeared that this was not so in a large series, and that in unilateral cases the right eye was attacked with approximately the same frequency as the left. Bilateral attacks occurred often as the epidemic became more protracted, sixteen cases being noted in the last 1,000 cases recorded in the year 1929, as compared with six cases in the first 923.

\section{Mistakes in Diagnosis}

The condition with which it was most likely to confuse superficial punctate keratitis, was simple catarrhal conjunctivitis. Cases of conjunctival hyperaemia with or without scanty discharge, which did not show any predominant organism in the smear, very frequently proved, when kept under corneal microscope observation, to be superficial punctate keratitis, and in due course the spots appeared on the cornea. A negative conjunctival smear, or a conjunctival smear within average limits, helped towards prognosticating the development of corneal lesions at a later date. It is obviously more difficult to diagnose the condition in eyes previously affected with any form of conjunctivitis. It was necessary in the out-patient department to follow up very carefully, cases of conjunctival hyperaemia, whether suspected to be due to organismal infection or simple forms of irritation. It was very easy to make mistakes with mild attacks; for example, a cataract case discharged on the fourteenth day showed a red eye some weeks later, the patient was promptly readmitted as it was considered likely that a post-operative complication had set in, but in a few days typical spots appeared on the cornea. It is unnecessary to detail all the possible conditions which might be confused with the milder types of the disease, particularly in the earlier stages, when the cornea is very slightly involved or not involved at all, but it may be stated that in a number of early cases the conjunctival hyperaemia was distinctly localised, being confined to perhaps a quadrant of the bulbar conjunctiva, and this was liable to give an impression of a mild phlyctenular condition, or localised irritant. Naturally the converse state of affairs was 
also true during the epidemic, and we were often too hasty in assuming that a simple catarrhal condition, or hyperaemic conjunctivitis due to irritants, or even a mild iritis with keratic precipitates when seen naked eye, was superficial punctate keratitis. Careful examination, however, of conjunctival smears, and of the cornea with the loupe and microscope, for the most part eliminated such sources of error. The lack of symptoms (irritation, lacrymation, photophobia) was a feature which helped the diagnosis when patients presented themselves early, but this very feature often kept them away till maculae appeared and they presented themselves for the first time with grey corneal spots and perhaps a dimness of vision, the previous redness being forgotten.

A few exceptional cases may be quoted. The first two illustrate difficulties of diagnosis in more severe attacks.

\section{Confusion with Familial Dystrophy}

A Hindu female attending the out-patient department, at first sight gave the impression of interstitial keratitis due to congenital syphilis, but on further examination there was nothing to support this view. The case was provisionally diagnosed as a severe bilateral superficial punctate keratitis. An indefinite history obscured a correct diagnosis. There were rounded, smooth, slightly elevated opacities arranged in an irregular ring around the pupillary area of the cornea, with a more diffuse haziness and fine grey dots both centrally and peripherally, though the extreme periphery was almost clear, and there was no vascularisation. The grey haze extended into the substantia propria, and through this zone one was able to see discrete grey deposits in the endothelium apparently similar to the elevated epithelial opacities, but smaller. It was the consideration of these endothelial opacities which made the provisional diagnosis doubtful, although the case resembled some rare forms of superficial punctate keratitis in which there had been no question as to the diagnosis. While we were still doubtful as to the nature of the case, two brothers called to take her away. The diagnosis was then obvious : all three had nodular dystrophy. The case was one of the familial bilateral affections of the cornea, which happened to be of the nodular type, associated with a diffuse haze and not so sharply confined to the centre of the cornea as others we have seen. In the younger brother alone was the sensibility of the cornea affected. Without an accurate family history it would have been difficult to make a diagnosis.

\section{Similarity to Conjunctivitis due to Irritants}

An adult male Muhammadan presented an appearance of intense redness of the bulbar conjunctiva on both sides with minute haemorrhages. The condition looked exactly like that sometimes seen in conjunctivitis induced intentionally by irritants. The 
symptoms were not so severe as the appearance warranted, nor did examination of the conjunctival smear point to a definite infection with pathogenic organisms. The corneal microscope showed typical minute corneal lesions.

\section{Unusual Type of Opacity}

D. M. M. arrived in Madras from England on October 21, 1929, and developed superficial punctate keratitis on December 23, 1929, in the right eye. In addition to the usual small discrete rounded corneal opacities, the superior temporal quadrant of the cornea near. the limbus showed a series of six horse-shoe shaped opacities, open towards the limbus. They appeared to cap the apices of vascular arcades. They slowly disappeared, as did the corneal opacities within six weeks.

\section{Further Observations on the Ophthalmoscopical Examination of the Posterior Segment for Pathological Changes}

A number of examinations were made in convalescent patients taken at random with negative findings in all cases.

\section{Late Examination of the Corneal Sensibility}

A number of observations showed that in cases recovered from the disease, the corneal sensibility was normal.

\section{Treatment}

Our ultimate experience of treatment was that atropine, and occlusion with a cold wet pad and bandage was preferable in the early stages; in the later stages dionine was most generally useful.

\section{Enlargement of the Preauricular Gland}

In two cases observed early in January, 1930, the patients first noticed a definite tenderness of the preauricular gland. These cases were otherwise exceptionally severe, in that chemosis was marked and there was considerable swelling of the lids. They were, however, undoubted cases of superficial punctate keratitis, in so far as the corneal appearances were concerned. Once our attention had been drawn to the possibility of enlargement of the preauricular gland, we were able to detect it in others. Six cases were noted in two months, during which period the epidemic was rapidly fading. This feature may have been missed earlier in the epidemic, but was undoubtedly absent in large numbers of cases of average intensity. It is difficult to estimate its significance. It may be due to the same agent which produces the corneal lesions or to a secondary infection. Against the latter supposition, it may be noted that in the cases quoted above, although they showed marked conjunctival hyperaemia and oedema, the bacteriological examination of the conjunctival sac was within average limits, 
that is to say, no characteristic or predominant organism was present.

\title{
Bacteriological Examination of the Conjunctival Sac
}

Col. King undertook further investigations in order to confirm our previous negative findings, and our thanks are due to him and to Dr. Sanjeva Rao, whom he placed in charge of the work. It seemed desirable that independent observers should confirm our failure to find such an organism as that described by Herbert in the Bombay epidemic. Up to date no organism has been seen or cultivated by them which might be regarded as aetiologically important, or identical with the organism described by Herbert. An attempt was made to devise an easy microscopical diagnostic method by taking scrapings from corneae showing early opacities, and staining smear preparations for inclusion bodies, but no simple practical method could be devised.

\section{TWO CASES OF GRANULOMA INVADING THE ORBIT DUE TO AN ASPERGILLUS}

BY

\author{
LT.-Col. R. E. Wright, C.I.E., M.D., I.M.S. \\ SUPERINTENDENT, GOVERNMENT OPHTHALMIC HOSPITAL, MADRAS \\ (Continued from The British Journal of Ophthalmology, \\ November, 1927)
}

At the time the two cases referred to in the title were published, it was not possible to give a definite position to the aspergillus concerned. It was mentioned, however, that Lt.-Col. H. W. Acton, I.M.S., Director, School of Tropical Medicine and Hygiene, Calcutta, hoped to work out the cultures originally isolated. He eventually forwarded the following note which completes the bacteriological investigation of these two rare and interesting cases.

\section{"Case I}

A. Material supplied (original tube) labelled 2 per cent. maltose, Asp. W. dated 2/10/1925.

B. A sub-culture from the original growth made by Lt.-Col. J. Cunningham, I.M.S., Pasteur Institute, Kasauli, to whom the original was supplied.

Both these tubes contained the Aspergillus Oryzae, characterized by the conidial heads being green in colour, the aerial hyphae pitted, the heads being globose and radiate shaped, sclerotica present, sterigmata in a single series. The conidia were colourless, smooth, and white, and the agar was uncoloured. 